\title{
Calibration of the RPC working voltage in the CMS experiment at the LHC
}

\section{Silvia Costantini*}

Department of Physics and Astronomy, University of Ghent, Ghent, Belgium

E-mail: Silvia.costantini@cern.ch

\section{On behalf of the CMS Collaboration}

The Resistive Plate Chambers (RPCs) are employed in the CMS experiment at the LHC as dedicated trigger system both in the barrel and in the endcap. The working voltage of all the RPCs installed in CMS has been tuned in 2011 with a dedicated High Voltage scan with LHC collisions during the low luminosity operations. This note presents procedures and results of the High Voltage calibration, with emphasis on the RPC detector uniformity and stability during the 2011 data taking period.

XI workshop on Resistive Plate Chambers and Related Detectors - RPC2012,

February 5-10, 2012

INFN Laboratori Nazionali di Frascati Italy

\footnotetext{
* Speaker.
} 


\section{The CMS experiment at the LHC}

The Large Hadron Collider (LHC) has become operational in 2009. High-energy physics runs took place in 2010 and 2011,with proton-proton collisions at a center-of-mass energy of 7 $\mathrm{TeV}$. The maximal instantaneous luminosity reached $3.5 \cdot 10^{33} \mathrm{~cm}^{-2} \mathrm{~s}^{-1}$ in 2011 . The Compact Muon Solenoid (CMS) [1], 2] Collaboration, one of the six experiments currently operating at the LHC, consists of over 3000 scientists, engineers and graduate students from 173 institutes in 40 Countries.

The central feature of the CMS detector is a superconducting solenoid, of $6 \mathrm{~m}$ internal diameter, providing a field of 3.8 T. Within the field volume are the silicon pixel and strip tracker, the lead-tungstate crystal electromagnetic calorimeter, and the brass-scintillator hadron calorimeter. Muons are measured in gas-ionization detectors embedded in the steel return yoke. In addition to the barrel and endcap detectors, CMS has extensive forward calorimetry, assuring very good hermeticity with pseudorapidity coverage up to high values $(|\eta|<5)$.

Muons with pseudorapidity in the range $|\eta|<2.4$ are measured with detection planes made of three technologies: Drift Tube chambers (DT), Cathode Strip Chambers (CSC) and Resistive Plate Chambers (RPC). Matching the muons to the tracks measured in the silicon tracker gives a transverse momentum $\left(\mathrm{p}_{\mathrm{T}}\right)$ resolution between $1 \%$ and $5 \%$, for $\mathrm{p}_{\mathrm{T}}$ values up to $1 \mathrm{TeV}$.

In 2011, CMS has recorded $5.20 \mathrm{fb}^{-1}$ of data out of $5.72 \mathrm{fb}^{-1}$ delivered by the LHC, for an efficiency of $91 \%$. Roughly $93 \%$ of the recorded data has been certified as "golden" for all physics analyses. An average of $98 \%$ of the subdetector channels are operational and in the readout.

\section{The CMS RPCs}

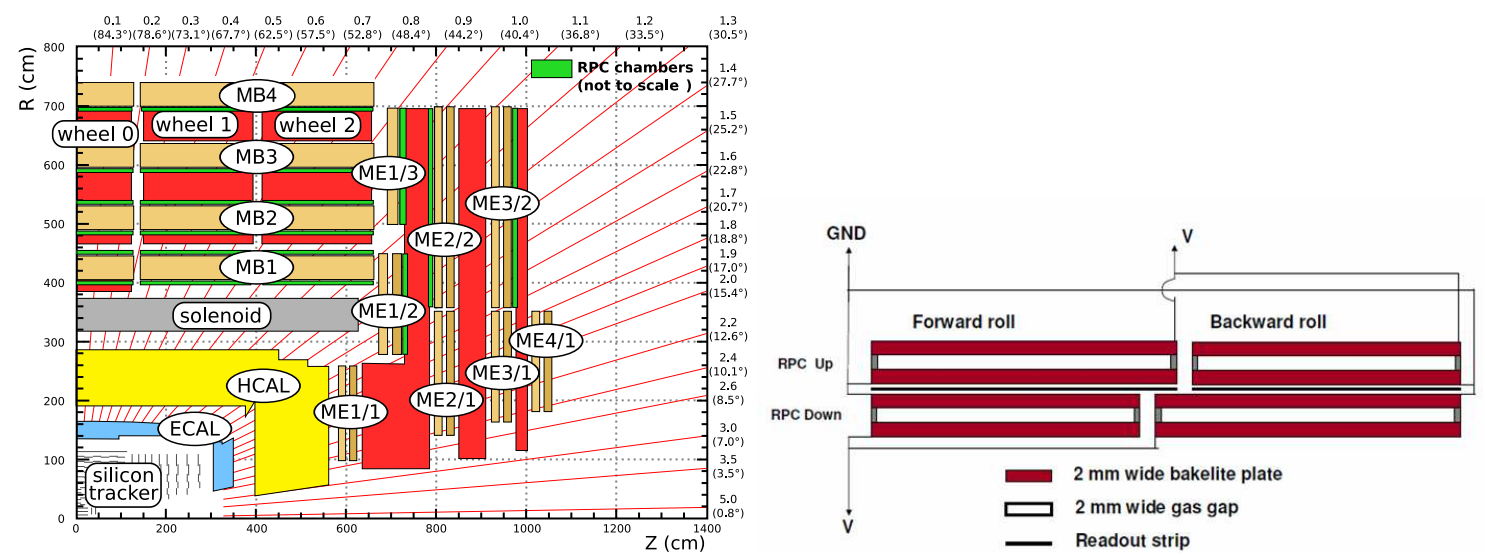

Figure 1: Left: Schematic view, in the R-z plane, of one quadrant of the CMS detector. The position of the RPC chambers is shown (not to scale). Right: Schematic layout of a double-gap barrel chamber composed by two sub-units, called rolls. The readout strip plane is also shown.

The RPC [3, 4] detectors are implemented in CMS as a dedicated trigger system, both in the barrel and in the endcap regions. Figure 1 shows a schematic view of one quarter of the CMS detector in the R-z plane (Fig. 1. left) and the layout of a double-gap barrel chamber (Fig. 1, right). Two gas gaps, of $2 \mathrm{~mm}$ each, are formed by two parallel bakelite electrodes, with one single plane of 
copper read-out strips in-between. The two gaps feature $2 \mathrm{~mm}$ thickness and have a bulk resistivity of the order of $10^{10} \Omega \mathrm{cm}$.

The barrel RPC system consists of five wheels, installed at $|\eta|<0.8$ and $|\mathrm{z}|<7 \mathrm{~m}$, subdivided into 12 azimuthal sectors, each one equipped with six radial layers of RPCs. Six endcap disks, three on the positive and three on the negative endcap side, are divided into 36 azimuthal sectors, with two radial rings in each one. They assure a full coverage up to $|\eta|<1.6$. In total, 480 barrel chambers and 432 endcap chambers are installed, adding up to 68136 barrel strips and 41472 endcap strips, respectively, covering a total surface of about $3000 \mathrm{~m}^{2}$. The CMS RPCs work in saturated avalanche mode and use a three-component, non-flammable gas mixture composed of 95.2\% $\mathrm{C}_{2} \mathrm{H}_{2} \mathrm{~F}_{4}$ (R134a), $4.5 \% \mathrm{iC}_{4} \mathrm{H}_{10}$ (isobutane) and $0.3 \% \mathrm{SF}_{6}$. Water vapor is added in order to maintain the relative humidity at constant values and allow for constant bakelite resistivity.

\section{Resolution and efficiency studies: the method}

The presence, next to each RPC chamber, of either a DT (in the barrel) or a CSC chamber (in the endcap), allows to profit by the redundancy of the CMS muon system in order to define the RPC hit efficiency independently of final physics "objects". Muon track segments, reconstructed in the multi-layer detectors DT and CSC, are linearly extrapolated to the RPC strip plane and used to predict RPC hits in a fiducial region around the extrapolated impact points, as illustrated in Figure 2 . Each extrapolated hit is matched to the closest RPC cluster, which is formed by contiguous fired strips. The typical cluster size corresponds to about two strips, as shown in Section 5 .

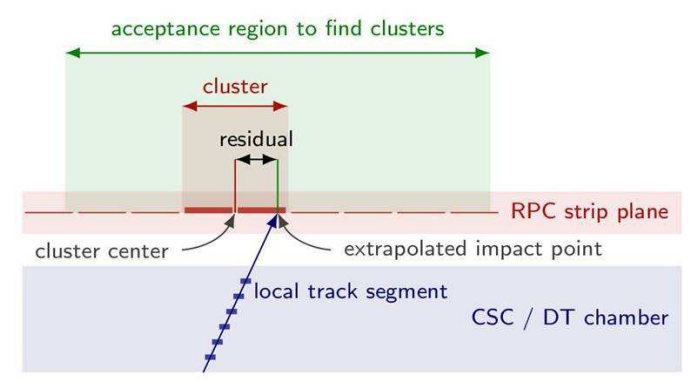

Figure 2: Schematic drawing of the extrapolation method. A DT or CSC track segment is linearly extrapolated to the RPC strip plane. RPC hits are sought in the acceptance region around the extrapolated impact point.

An additional requirement is applied to ensure that only extrapolated segments associated to muon tracks with hits in the central tracker are taken into account, while DT/CSC segments probably originated by neutral background particles are discarded. The three muon detectors employ different technologies and materials, thus they have different sensitivities to the various backgrounds.

The extrapolation method allows to measure both the RPC spatial resolution, through the residuals, and the hit efficiency. The measured spatial resolution increases with increasing strip widths and it ranges from $0.8 \mathrm{~cm}$ (for inner detector layers, characterized by smaller strip pitches) to $1.3 \mathrm{~cm}$ (for outer layers), both in the Barrel and in the Endcap. The strip pitch dimensions are between 2.28 and $4.10 \mathrm{~cm}$ in the barrel and between 1.95 and $3.63 \mathrm{~cm}$ in the endcap. 


\section{The High Voltage Scan. Detector uniformity}

A High Voltage (HV) scan was performed at the beginning of the 2011 proton-proton LHC running, aiming at defining the optimal operating HV for each individual chamber. The variation of the environmental pressure $\mathrm{P}$ and the temperature $\mathrm{T}$ inside the CMS cavern was taken into account as described in Section 5 .

Collision data were taken at eleven different values of the effective $\mathrm{HV}\left(\mathrm{HV}_{\text {eff }}\right.$, defined in Eq. (5.1) below), from $8.5 \mathrm{kV}$ to $9.7 \mathrm{kV}$. It is worth mentioning that in 2011 only a negligible amount of data (about $3 \mathrm{pb}^{-1}$ out of $5.72 \mathrm{fb}^{-1}$ ) was discarded, i.e. not included in the "golden" sample for physics analyses, because of the RPC HV calibration. Each data point needed roughly 30 minutes of low-luminosity $\left(10^{30}-10^{31} \mathrm{~cm}^{-2} \mathrm{~s}^{-1}\right)$ collision data. A dedicated data stream was used, containing information from the muon detectors and the first level trigger.

The efficiency $(\varepsilon)$ dependence on $\mathrm{HV}_{\text {eff }}$ is parametrized by a sigmoidal response function that can be written as:

$$
\varepsilon=\frac{\varepsilon_{\max }}{1+\mathrm{e}^{-\lambda\left(\mathrm{HV}_{\mathrm{eff}}-\mathrm{HV}_{50 \%}\right)}}
$$

where $\varepsilon_{\max }$ is the asymptotic efficiency for $\mathrm{HV} \rightarrow \infty$, the $\lambda$ coefficient is proportional to the sigmoid slope at the inflection point, and the High Voltage value $\mathrm{HV}_{50 \%}$ is the inflection point of the sigmoid, for which $50 \%$ of $\varepsilon_{\max }$ is reached.
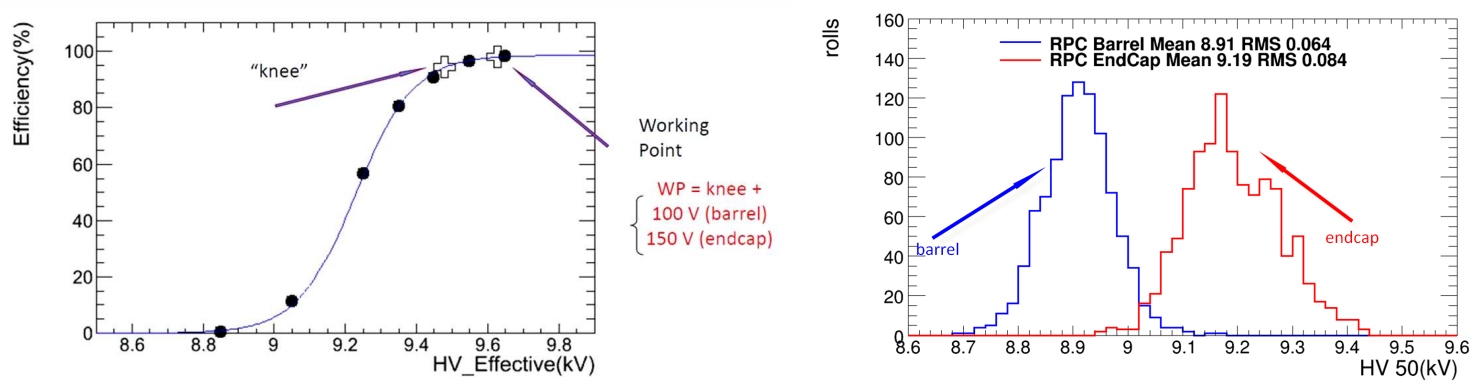

Figure 3: Left: efficiency measurement (in \%), as a function of the effective High Voltage $\mathrm{HV}_{\text {eff }}$ (in $\mathrm{kV}$ ) for a typical chamber. The "knee" and the optimal working point, shown in the picture, are defined in the text. Right: $\mathrm{HV}_{50 \%}$ (in $\mathrm{kV}$ ) distribution for the barrel (left curve, blue) and the endcap (right curve, red) rolls. $\mathrm{HV}_{50 \%}$ is the inflection point of the sigmoid, for which 50\% of the asymptotic efficiency is obtained.

Figure 3 (left) shows the efficiency as a function of the effective High Voltage for a typical endcap chamber. The "knee" is the $\mathrm{HV}_{\text {eff }}$ value for which $95 \%$ of the asymptotic efficiency is reached. The optimal working point (WP) is chosen beyond the knee, to ensure high efficiency, and in the plateau region, to minimize the dependence on the environmental parameters. The WP is then defined for each individual chamber as the knee value plus $100 \mathrm{~V}$ (for barrel chambers) or $150 \mathrm{~V}$ (for endcap chambers). The difference between barrel and endcap reflects the different trigger algorithms. The endcap trigger algorithm, requiring three coincidences out of three planes, is more sensitive to efficiency variations. The resulting WP values are averaged for chambers fed by the same HV supplier.

The results of the sigmoidal fit are highlighted in Figure 3 (right), presenting the $\mathrm{HV}_{50 \%}$ distribution for barrel and endcap. The different $\mathrm{HV}_{50 \%}$ average values (around $8.9 \mathrm{kV}$ for the barrel 
and $9.2 \mathrm{kV}$ for the endcap) might be due to different construction techniques. As shown in Figure 3 (right), a high level of uniformity is obtained both for the barrel and for the endcap chambers, with RMS values of the $\mathrm{HV}_{50 \%}$ distributions of the order of $60 \mathrm{~V}$ and $80 \mathrm{~V}$, respectively.

\section{Detector stability}

The variation of the environmental pressure $\mathrm{P}$ and the temperature $\mathrm{T}$ inside the CMS cavern was taken into account by rescaling [5] to the chosen reference values $\left(\mathrm{P}_{0}=965 \mathrm{mbar}\right.$ and $\mathrm{T}_{0}=$ $293 \mathrm{~K})$ :

$$
\mathrm{HV}_{\mathrm{eff}}(\mathrm{P}, \mathrm{T})=\mathrm{HV} \cdot \frac{\mathrm{P}_{0}}{\mathrm{P}} \cdot \frac{\mathrm{T}}{\mathrm{T}_{0}},
$$

where $\mathrm{HV}_{\text {eff }}$ is the resulting effective $\mathrm{HV}$. The dominant effect is due to the pressure variation: a $1 \%$ variation, of the order of 10 mbar, produces a sizeable $\mathrm{HV}_{\text {eff }}(\mathrm{P}, \mathrm{T})$ difference of about $100 \mathrm{~V}$. Starting from July 2011, the $\mathrm{HV}_{\text {eff }}(\mathrm{P}, \mathrm{T})$ correction of Eq. (5.1) was automatically implemented.

Figure 4 and Figure 5 show preliminary results obtained with 2011 data. The efficiency and the cluster size stability as a function of time have improved after the introduction of the automatic $\mathrm{HV}_{\text {eff }}(\mathrm{P}, \mathrm{T})$ correction of Eq. (5.1). The barrel efficiency fluctuations (Fig $甘$, left), mainly due to pressure variations in the CMS cavern, are reduced from about $\pm 1 \%$ to about $\pm 0.5 \%$. The higher average efficiency ( $\sim 97 \%$ compared to $\sim 96 \%$ ) in the first part of 2011 is due to the choice of 965 mbar as a reference value for rescaling. In the first part of the year, (Fig 4 ) the atmospheric pressure was on average lower than 965 mbar, giving rise to higher $\mathrm{HV}_{\text {eff }}$ values and therefore to higher efficiency values with respect to the second part of the year.

An increased stability, with reduced fluctuations, is also observed in the endcap cluster size as a function of time (Fig 4 , right). As mentioned above for the efficiency, the same considerations apply to the average values before and after the $\mathrm{HV}_{\text {eff }}(\mathrm{P}, \mathrm{T})$ correction.

Fig 5 presents the average cluster size in the barrel (left) and in the endcap (right) as a function of the atmospheric pressure, before and after the automatic correction. Both in the barrel and in the endcap, a clear anti-correlation is shown in the first part of 2011, when the correction was not applied. The slight positive correlation in the second part of the year might hint at an overcorrection in Eq. (5.1), currently under study.

\section{Conclusions}

The 2011 HV scan has been extremely effective, allowing to select the optimal operating HV values for each individual RPC chamber and to obtain a high level of uniformity both in the barrel and in the endcap. A new method for determining the RPC hit efficiency is used by the CMS RPC Collaboration and automatic $\mathrm{HV}_{\text {eff }}(\mathrm{P}, \mathrm{T})$ corrections are in place since July 2011. Those efforts result in increased efficiency stability and increased cluster size stability as a function of pressure and time. The HV scan is going to be performed again at the beginning of the 2012 data taking period. 

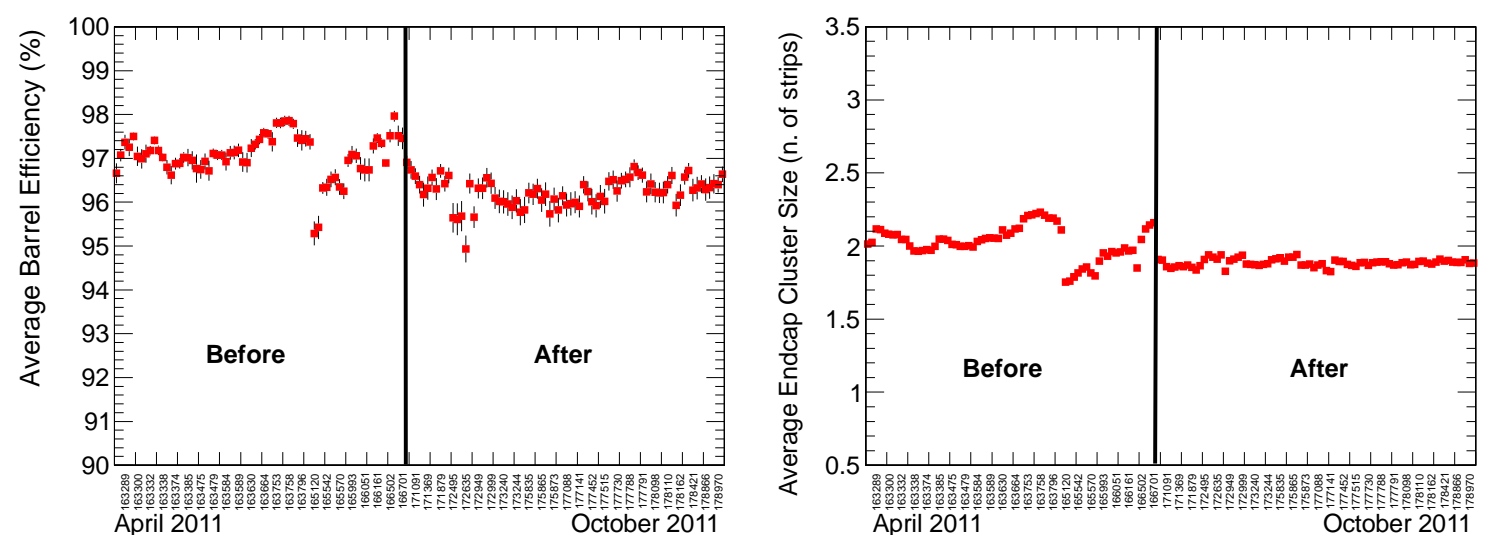

Figure 4: Left: Barrel efficiency (in \%) as a function of the run number, i.e. as a function of time, for runs taken between April 2011 and October 2011. The two regions, before and after the automatic $\mathrm{HV}_{\mathrm{eff}}(\mathrm{P}, \mathrm{T})$ correction, are shown in the plot. Right: Endcap cluster size (in number of strips), as a function of the run number, for the same run range between April and October 2011.
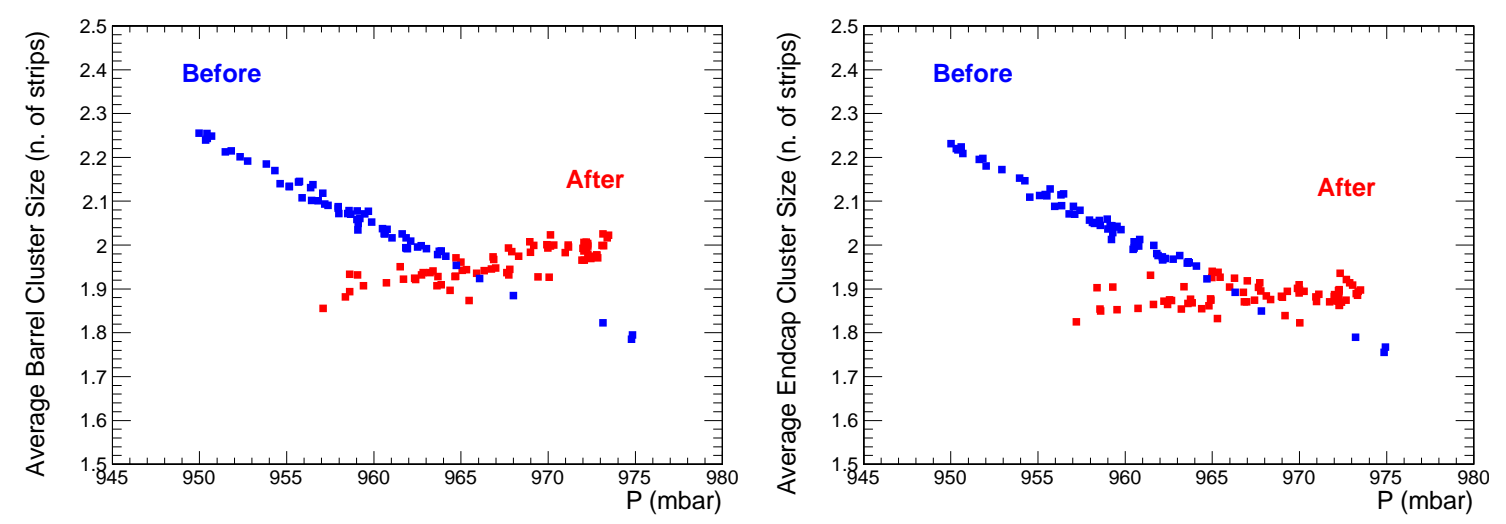

Figure 5: Average cluster size for the barrel (left) and for the endcap (right), in number of strips, as a function of the atmospheric pressure P (in mbar), for runs before (left, blue curve) or after (right, red curve) the automatic $\mathrm{HV}_{\text {eff }}(\mathrm{P}, \mathrm{T})$ correction.

\section{References}

[1] CMS Collaboration, "The Compact Muon Solenoid”, Technical Proposal, CERN/LHCC 94-38 (1994).

[2] CMS Collaboration, "The CMS Experiment at the CERN LHC”, JINST 3 (2008) S08004, http://iopscience.iop.org/1748-0221/3/08/S08004.

[3] M. Abbrescia et al., "Cosmic ray tests of double-gap resistive plate chambers for the CMS experiment”, Nucl. Instrum. Meth. A 550 (2005) 116, doi: 10.1016/j.nima.2005.06.074.

[4] CMS Collaboration, "Performance Study of the CMS Barrel Resistive Plate Chambers with Cosmic Rays”, JINST 5 (2010) T03017, doi: 10.1088/1748-0221/5/03/T03017 and references therein.

[5] M. Abbrescia et al., "Resistive plate chambers performances at cosmic rays fluxes", Nucl. Instrum. Meth. A 359 (1995) 603. 